\title{
Erratum to: What could be caused must actually be caused
}

\section{Christopher Gregory Weaver}

\section{Erratum to: Synthese \\ DOI: 10.1007/s11229-010-9814-3}

On page 15 of the published article after the following sentence, there should have been a reference to footnote 47 :

"But, the quantum state attributed to the system allows one to infer that a given one of these outcomes will arise with a certain probability (see footnote 47)."

On page 15 of the published article after the following sentence, there should have been a footnote:

"The Kochen proof attempted to show that, the interrelationship among measured values predicted by quantum mechanics, are incompatible with any possibility of the values being fully determined by underlying values of hidden parameters. ${ }^{1}$

Thanks to Lawrence Sklar (via email correspondence) for being so nice in encouraging me not to worry about these accidental omissions. Note: These subtle omissions occurred accidently during the proof correction process.

\footnotetext{
${ }^{1}$ Sklar (1992, p. 212).
}

The online version of the original article can be found under doi:10.1007/s11229-010-9814-3. 